\title{
EDITORIAL
}

\section{LA SALUd PÚBLICA EN EL Perú y el COVID-19}

\author{
Public health in Peru and COVID-19
}

Jesús Lorenzo Chirinos-Cáceres ${ }^{1}$

त pesar de haber mejorado el control de enfermedades que azotaban a los países de ingresos bajos y medios, cada clerto tiempo aparecen nuevas enfermedades infecciosas, que también comprometen a los países de ingresos altos, haciéndoles recordar que las transiciones demográficas y epidemiológicas son dinámicas y cambiantes por lo que debíamos estar atentos y preparados. ${ }^{1}$

Para todos nosotros no es ajeno haber conseguido logros como el incremento en la esperanza de vida al nacer, disminución de la mortalidad materna e infantil y control de las enfermedades metaxénicas, a pesar de sus rebrotes, entre otros indicadores. El progreso en el control de nuestros problemas de salud, así como en los sistemas de salud, ha sido desigual $y$, en varios casos, manchado por la corrupción. ${ }^{2,3}$

Los estilos de vida saludable han tenido una relación inversa al progreso de las economías por el incremento del sedentarismo y el uso y abuso de la tecnología en los espacios privados y sociales, los que han representado factores importantes para el incremento de enfermedades crónicas como la obesidad, diabetes mellitus, hipertensión esencial, etc.,

1 Decano de la Facultad de Salud Pública, Universidad Peruana Cayetano Heredia, Lima, Perú. con los respectivos daños cardiovasculares y el incremento del cáncer.

Ha habido poco desarrollo de la atención primaria y cierto abandono en la atención hospitalaria llegando a la precariedad y a dificultar el acceso al sistema de salud a pesar de la insistencia en la universalización de la salud y en los Objetivos de Desarrollo Sostenible para salud y bienestar (ODS 3), que en varios casos encontraba un sistema sumamente fraccionado y para nada integrado.4 "La meta específica de no dejar a nadie desatendido es una llamada de atención para centrarse en las personas que corren más riesgo de quedarse sin los servicios de salud que necesitan y merecen" es un plan al 2030. ${ }^{1}$

Así mismo, debemos señalar algunos aspectos de la educación ya propuestos el siglo pasado en la rueda y la taxonomía de Bloom que indican que la educación de calidad (ODS 4) debería ir de un nivel de complejidad bajo a un nivel de complejidad alto mediante el conocimiento, comprensión, aplicación, análisis, síntesis y evaluación, por supuesto todo dentro de lo familiar, comunitario, ambiental, regional, nacional y global en que se da la vida. ${ }^{5}$

Es importante mencionar que la salud y la educación (ODS 3 y 4) deben ir juntas en todas 
las etapas de la vida por ser transversales a todas las demás ODS y poder tener una mente abierta para hacer frente a los vicios y virtudes del sistema en general.

Todo lo anterior sirve para indicar que la salud pública, en una definición amplia, debería ser entendida como la ciencia y el arte de promover, prevenir, controlar e impedir enfermedades, mejorando la calidad y aumentando la esperanza de vida, fomentando una salud de las poblaciones de manera equitativa, de acceso libre y permanente a un sistema de salud integrado o único y universal, con participación y fiscalización comunitaria, con un medio ambiente saludable, con educación sanitaria e higiene personal que lleven al autocuidado en salud. ${ }^{6}$ Fue difícil encontrar una definición pero, con esta, pretendo señalar lo ocurrido en estos cuatro meses de pandemia. Empezaré indicando que la epidemiología y la salud pública permiten promover salud y prevenir enfermedades haciendo predicciones con los patrones de enfermedades ya estudiadas, basados en su nivel más bajo en factores asociados, luego factores de riesgo, factores esenciales y los determinantes sociales. Todo ello debe llevar a políticas tanto en salud como en educación con una visión multimodal, multisectorial e interdisciplinaria.

Sólo mencionaré algunos ejemplos de mi experiencia como médico internista, de haber asistido a estudios epidemiológicos como infecciones nosocomiales o intrahospitalarias, que cuestan tanto a los pacientes y hospitales. Luego, sobre mortalidad materna, cuyos factores de riesgo clínicos más importantes eran la hemorragia y las infecciones y su relación con el aborto, donde ya entran factores sociales, demográficos y económicos, como la pobreza, educación, grupo etario, etc. La epidemia del VIH/SIDA, el cólera, la influenza H1N1, la resistencia bacteriana, etc. Sin embargo, he apreciado que todas estas experiencias no nos han permitido avanzar en el sistema de salud y educación correspondientes ante epidemias mayores.

La historia se repite y tiene relación con nuestra situación actual de la educación y salud y la falta de comunicación para el cambio de comportamientos. La salud y la educación han servido para lograr ocupar cargos en la política más que solucionar sus problemas; y, en esta pandemia se ha puesto al descubierto todas las falencias de nuestros sistemas de salud y educativo, por mencionar dos de suma importancia.

A mediados de los años 1990`s, en la Oficina General de Epidemiología con el Programa de Epidemiología de Campo, una regla era leer los diarios porque allí se encontraban señales de inicios de brotes epidémicos y se podían revisar los supuestos de las comunidades sobre el origen de las mismas. Todo ello permitía prepararse para los brotes epidémicos y alertar a las comunidades y al sistema de salud, y con ello se pudo yugular brotes de malaria por Plasmodium falciparum, peste y rabia. Sin embargo, nada de ello, permitió mejorar la educación sanitaria e higiene ni integrar, por lo menos, el sistema de salud.

En China, en diciembre 2019 (al parecer hubo casos antes) apareció el brote del COVID-19, producido por el SARS-CoV-2, y en el Perú apareció el primer caso el 6 de marzo del 2020 y se decidió el cierre de las actividades en el país el 16 marzo. ${ }^{7}$ Pero, no se tomaron otras medidas importantes previas como revisar a los pasajeros que llegaban del extranjero al país, sobre todo de países comprometidos, o limitar su entrada, el cierre del aeropuerto, suspender los viajes a las regiones y adoptar todas las medidas de autocuidado conocidas como lavado de manos, el uso de mascarillas (que recién dos meses después se generalizó), 
el distanciamiento físico y el aislamiento social $\mathrm{y}$, muy importante, proteger al personal de salud.

Peor aún, la protección del personal de salud fue escasa, se usaron pocas pruebas diagnósticas certeras y más las complementarias, se tomaron medidas que rompieron el aislamiento social y el distanciamiento físico llevando a un incremento exponencial de casos que aún no para. Así, estando en el día 141 del estado de emergencia nacional (según el mapa interactivo del Centro Johns Hopkins de Ciencia e Ingeniería de Sistemas), al 31 de julio 2020, el Perú tenía 428850 casos y 19614 fallecidos que significaban la mayor cantidad de muertos por millón de personas en el mundo. Y, que hubo momentos en que se dijo que se había controlado la enfermedad o que habíamos llegado a una meseta que era irregular o con picos y en la actualidad estamos ante un repunte al haberse establecido el reinicio de las actividades económicas por fases y los viajes interprovinciales por vía aérea y terrestre, que son vías de infección importantes, y con una cuarentena focalizada incipiente o inexistente. Además, se iniciaron tratamientos no comprobados o automedicaciones, con solo supuestas eficacias, y no sabemos si los fallecimientos ocurrieron por la enfermedad y sus complicaciones o por otros eventos.

También, debemos señalar que, aparte de la contribución hecha por el gobierno de turno ante esta situación, hay corresponsabilidad de las autoridades locales y regionales, así como de la misma población en su desesperación por conseguir alimentos, medios económicos y tratamientos de dudosa eficacia debido principalmente a los determinantes sociales de vivienda, educación, salud y pobreza que son precarios.
Podríamos haber predicho que la situación del COVID-19, en las condiciones de salud pública y de educación en que nos encontrábamos, iba a sobrepasar toda acción tomada por el gobierno. Así, la realidad brusca y violenta fue mostrada por esta enfermedad, produciendo cambios, aparte de su efecto negativo en la economía, en la educación en el país, como la educación a distancia que es incompleta $\mathrm{y}$, en cuanto a la salud, mostrándonos la falta de oxígeno, de camas hospitalarias para los sintomáticos moderados, de camas en las unidades de cuidados intensivos, de ventiladores mecánicos y de recursos humanos especializados para los más comprometidos (UCI), llegándose a la situación de que los médicos tienen que decidir quién entra a UCI y recibe soporte ventilatorio, aparte del sufrimiento de los pacientes de saber que van a fallecer solos y en condiciones deplorables.

Lo violento de esta enfermedad hace que nos pongamos a tomar acciones prontas y equitativas, con una integración inicial del sistema de salud, las cuales ya ha sido dadas a conocer por los expertos, y seguir las normas establecidas para preservar la salud. Finalmente, debe prevalecer la lucha contra la corrupción y planear una política de desarrollo sostenido que permita que los peruanos podamos estar en mejores condiciones para enfrentar nuevas emergencias sanitarias, así como las secuelas que dejará el COVID-9 en la salud de las personas, teniendo como corolario que "educación sin salud no es educación y salud sin educación tampoco es salud".

Debo indicar que, de acuerdo a la definición de salud pública utilizada, hay mucho que reflexionar y hacer, y nada que resaltar al momento actual, hasta que llegue la vacuna o el tratamiento salvador con evidencias suficientes. 


\section{REFERENCIAS BibliográficAs}

1. Amina JM, Tedros AG. Vida saludable, bienestar y objetivos de desarrollo sostenible. (editorial) Bulletin of the World Health Organization 2018;96:590-590A. URL disponible en: https:// www.who.int/bulletin/volumes/96/9/18-222042/es/ (Fecha de acceso:3/8/2020).

2. García PJ. Lecture: Corruption in global health: the open secret. The Lancet/The Academic Medical Sciences. Published Online November 27, 2019 https://doi.org/10.1016/ S01406736(19)32527-9 www.thelancet.com. URL disponible en: http: / / dx.doi.org/10.1016/S0140-6736(19)32527-9

3. Chirinos-Cáceres, JL. La corrupción en el sector salud: ¿A quién afecta? Rev Soc Peru Med Interna. 2019;32(4): 125-126 . https:/ / doi.org/10.36393/spmi.v32i4.489.
4. Chirinos-Cáceres JL. COVID-19: La salud en el Perú en tiempos del coronavirus: A reflexionar. Rev Soc Peru Med Interna. 2019;33(1):10-11. https://doi.org/10.36393/spmi. v33i1.504

5. Bloom, Benjamin. (2020, 22 de junio). Wikipedia, La enciclopedia libre. URL disponible en: https://es.wikipedia.org/w/index. php?title=Benjamin_Bloom\&oldid=127158613 (Fecha de acceso: 03-08-2020).

6. La filosofía de la salud pública. Revista Cubana de Salud Pública. 2014;40(1):144-158. URL disponible en: https://scielosp.org/ $\mathrm{pdf} / \mathrm{rcsp} / 2014 . \mathrm{v} 40 \mathrm{n} 1 / 144-158$

7. Chirinos-Cáceres, J. La salud en el Perú en tiempos del coronavirus: 60 días después, ¿sin reflexión? Rev Soc Peru Med Interna. 2019;33(2): 56-57. URL disponible en: https:/ / doi. org / $10.36393 /$ spmi.v33i2.520

FECHA DE RECEPCIÓN: 17-08-2020.

FECHA DE ACEPTACIÓN: 30-08-2020. 